\title{
Exploring the Relationship among Dimensions of Flight Comprehensive Capabilities Based on SEM
}

\author{
Ruishan Sun and Yang Li \\ Research Institute of Civil Aviation Safety, Civil Aviation University of China, \\ Tianjin, 300300, China \\ sunrsh@hotmail.com
}

\begin{abstract}
The structural equation model is constructed to explore the relationship among basic cognitive abilities, personality traits and mental health of pilots. A framework of hypotheses is established to test the relationship among the three dimensions based on theories in the literature. Data is gathered from 65 pilots using 3 questionnaires. The model shows that both personality traits and mental health can affect the cognitive functions significantly, and that emotion characteristic, character traits and working attitude will also have an impact on a pilot's basic cognitive ability by affecting his mental health state. The results suggest that not only flight cognitive abilities but also personality traits and mental health can affect a pilot in terms of flight performance.
\end{abstract}

Keywords: flight comprehensive capability, flight aptitude, personality traits, mental health, SEM.

\section{Introduction}

Since the early days of aviation history, cognitive and psychomotor skills have been seen as important aspects of being a good pilot. Moreover, personality traits of pilots have also attracted many researchers' attention. Research strategies such as observation of pilots and participant observation have been used to examine which personality traits are important in pilot selection. In particular, dated back to 1921, Dockeray and Isaacs concluded in their study on psychology in aviation that "quiet methodical men were among the best flyers" because of their "power and quick adjustment to a new situation and good judgment" [1]. In 1950s, Saul Sell and his colleagues led a project in the United States to find suitable personality measure for pilot selection. They evaluated a total of 26 personality measures and found that personality tests were better predictors of long-term criteria compared to ability tests[2][3]. With the introduction of computers in testing, a number of new personality-related concepts have also been evaluated on pilots, including measures of risk taking, assertiveness, field dependency, and attitudes [4]. Mental health is another topic that has so far been emphasized in some degrees during the evaluation process on pilots. To meet social demands or solve work-related tasks, the individual relies on different sets of resources, including knowledge, experience, and personal attributes [5]. Some theories describe mental problems as the 
result of factors or elements that have a negative impact on the individual. Other theories are concerned with stress, negative emotion and physical reactions.

However, few studies on the relationship among cognitive abilities, personality traits and mental health in aviation can be found in the literature. This paper tries to fill this gap and proposes to study the relationship among the three dimensions based on the structural equation model (SEM).

\section{Theory and Hypotheses}

\subsection{Research Structure of Flight Comprehensive Capabilities}

The concept of flight comprehensive capabilities is the core of flight abilities. It can be defined broadly as every internal factor that contributes to consistent behavior in different situations or, narrowly, as encompassing only cognitive functions, personality, emotions and motivation. In this study we build up a system of flight comprehensive capabilities with three dimensions including basic flight cognitive ability, personality traits and mental health. Basic flight cognitive ability is defined as a cognitive process of acquiring, processing, memorizing and applying information, including attention, working memory, judgment and decision making, speech comprehension, logical reasoning and spatial cognitive ability. Personality traits are analyzed by a matrix of character traits, emotion traits, intellect and working attitude. Character traits are personal notions that describe how a person appears to others. Emotion traits are described as emotional stability and mental experience. Intellect is a capacity for rational thought or inference or discrimination, and a willingness to pursue and explore the unfamiliar experience. Working attitude reflects individual achievement motivation, responsibility sense and self-discipline. Mental health can be defined as the psychological state of a pilot who is functioning at a satisfactory level of emotional and behavioral adjustment, which reflects his emotion, level of stress and adaptability. This paper will discuss mental health from these three aspects.

\subsection{Theory and Hypotheses}

A. Relationship between personality traits and flight cognitive ability. Personality traits play an important role in explaining a pilot's behavior. Pilots with certain personality characteristics such as achievement motivation and emotional stability have better flight performance. Early studies also showed that intellect reflects an individual's level of cognitive abilities, and therefore is very important. Moreover, experimental results showed that those who had the most positive attitudes towards their job performed slightly better [6]. Base on these arguments, we establish several hypotheses on the relationship between personality traits and flight cognitive abilities, as shown in Table 1.

B. Relationship between mental health and flight cognitive abilities. Mental health has a direct effect on an individual's job performance. A critical stress situation may 
arise when something unusual takes place during a flight. Examples of stress situations include an indication of technical difficulties or a rapidly deteriorating weather condition. Moreover, Bad interpersonal relationship or work-life conflicts may also result in a negative emotion. It is important to analyze typical reactions in such situations, be aware of how the crew responds to stress and negative emotion, and understand how it affects decisions made during the flight. According to the review provided by Orasanu [7], mental issues such as stress, depression, anxiety and so on, may have the following effects: (1) people make more errors; (2) attention is reduced; (3) working memory is reduced; (4) change of strategy: speed gains preference to accuracy. Thus, cognitive functions are subject to a number of stress-related consequences in terms of how we perceive our surroundings, process information, and make decisions. Table 2 shows hypotheses that we would like to test on the relationship between mental health and flight cognitive abilities.

C. Relationship between personality traits and mental health. There is little doubt that some working environments are generally considered stressful. However, people with certain personality characteristics experience stress or negative emotion more often and more intensely, or have stronger adaptability than others. Studies have shown that many organizations tend to reward certain types of workers, who are characterized by irritability, aggression, hostility, and ambition, because these factors usually indicate a personality of achievement striving. However, on the other hand, these personality characteristics may cause emotional problems for the individual and his or her surroundings such as stress and burnout. Furthermore, people with active coping techniques (i.e., who act strategically to handle difficult situations) generally score lower on burnout than people who use more emotion-focused coping techniques (e.g., the person attempts to deal with emotions by seeking comfort in other people) [5]. Earlier studies have found that stress can be triggered also by high interpersonal demands; while later studies have shown that individual difference is an important factor as well. Based on these studies, we build up the hypotheses on the relationship between personality traits and mental health, as shown in Table 3.

Table 1. Hypotheses on the relationship between personality traits and flight cognitive ability

\begin{tabular}{cl}
\hline H\# & \multicolumn{1}{c}{ Hypotheses } \\
\hline H1 & Intellect has a direct positive relationship with spatial imagination \& image-thinking \\
H2 & Intellect has a direct positive relationship with speech comprehension \\
H3 & Intellect has a direct positive relationship with spatial cognitive \& logical reasoning \\
H4 & Intellect has a direct positive relationship with spatial perception ability \\
H5 & Intellect has a direct positive relationship with judgment \& decision making \\
H6 & Intellect has a direct positive relationship with working memory \& attention. \\
H7 & Character traits has a direct positive relationship with speech comprehension \\
H8 & Character traits has a direct positive relationship with judgment \& decision making \\
H9 & Working attitude has a direct positive relationship with spatial imagination \\
H10 & Working attitude has a direct positive relationship with speech comprehension \\
\hline
\end{tabular}


Table 2. Hypotheses on the relationship between mental health and flight cognitive ability

\begin{tabular}{ll}
\hline H\# & \multicolumn{1}{c}{ Hypotheses } \\
\hline H11 & $\begin{array}{l}\text { Adaptability has a direct negative relationship with spatial imagination \& } \\
\text { image-thinking }\end{array}$ \\
H12 & $\begin{array}{l}\text { Adaptability has a direct negative relationship with speech comprehension } \\
\text { Adaptability has a direct negative relationship with spatial cognitive \& logical } \\
\text { H13 }\end{array}$ \\
reasoning & Adaptability has a direct negative relationship with space perception ability \\
H15 & Adaptability has a direct negative relationship with judgment \& decision making \\
H16 & $\begin{array}{l}\text { Adaptability has a direct negative relationship with working memory and attention } \\
\text { H17 }\end{array}$ \\
Stress situation has a direct negative relationship with spatial imagination \& \\
H18
\end{tabular}

Table 3. Hypotheses on the relationship between personality traits and mental health

\begin{tabular}{cl}
\hline H\# & \multicolumn{1}{c}{ Hypotheses } \\
\hline H24 & Emotion characteristics has a direct negative relationship with adaptability \\
H25 & Character traits has a direct negative relationship with adaptability \\
H26 & Working attitude has a direct negative relationship with adaptability \\
H27 & Emotion characteristics has a direct negative relationship with emotional health \\
H28 & Working attitude has a direct negative relationship with stress situation \\
\hline
\end{tabular}

As a summary, Fig. 1 presents the theoretical framework of all the above hypotheses on the relationship among flight cognitive abilities, personality traits, and mental health.

\section{Data Gathering}

The structural equation model (SEM) is a statistical technique for testing and estimating causal relations using a combination of statistical data and qualitative causal assumptions [8]. We distributed a packet of 3 questionnaires to 65 pilots aged 20 to 60 years old. The three questionnaires are Flight Cognitive Capability Test, the Symptom Checklist-90 (SCL-90), and the Sixteen Personality Factor Questionnaire (16PF). Then the theoretical model was established to explore the relationship among basic flight cognitive ability, personality traits and mental health. Latent variables and manifest variables in the SEM are shown in Table 4. 


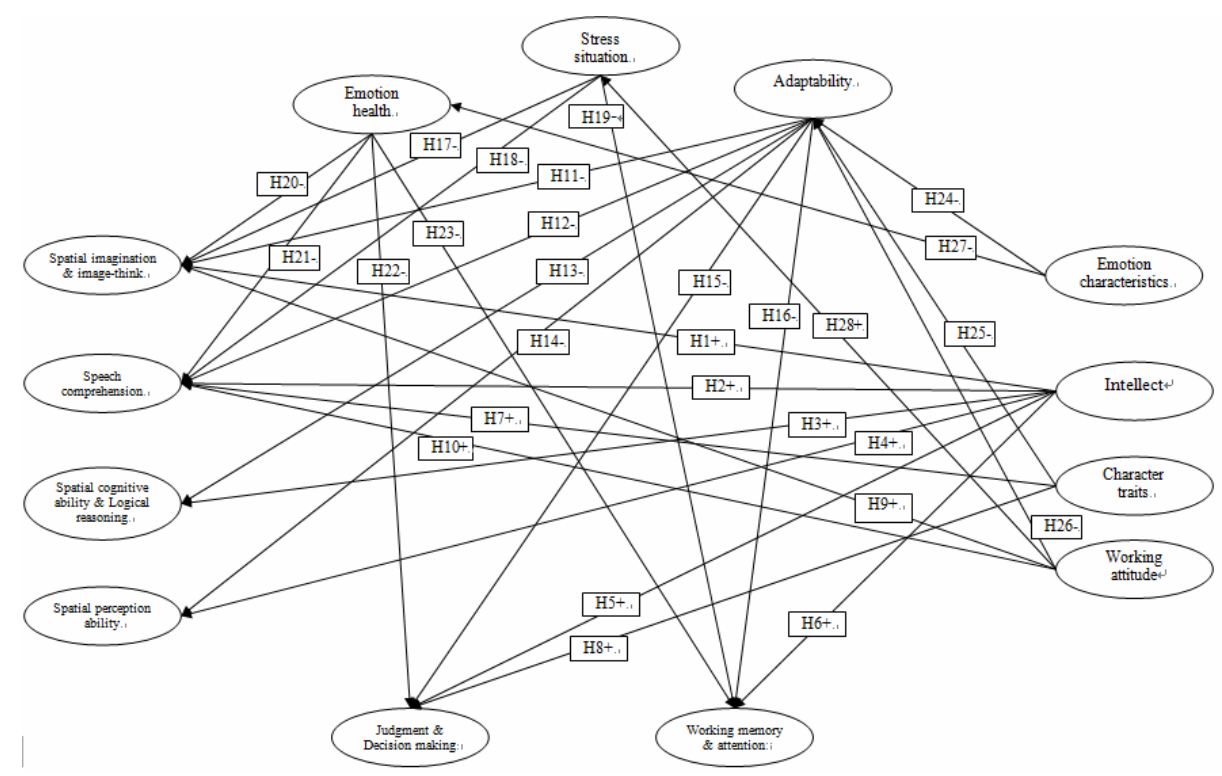

Fig. 1. Theoretical hypotheses of the study

Table 4. Latent variables and manifest variables

\begin{tabular}{|c|c|}
\hline Latent variables & Manifest variables \\
\hline Emotion characteristics $(\mathrm{P} 1)$ & $\begin{array}{l}\text { Emotional Stability, Apprehension, } \\
\text { Liveliness, Tension }\end{array}$ \\
\hline Intellect (P2) & $\begin{array}{l}\text { Reasoning, Abstractedness, Openness to } \\
\text { Change }\end{array}$ \\
\hline Character traits $(\mathrm{P} 3)$ & Warmth, Dominance, Vigilance, Privateness \\
\hline Working attitude (P4) & $\begin{array}{l}\text { Rule-Consciousness, Social Boldness, } \\
\text { Sensitivity, Self-Reliance, Perfectionism }\end{array}$ \\
\hline $\begin{array}{l}\text { Spatial imagination \& image-thinking (N1) } \\
\text { Speech comprehension (N2) } \\
\text { Spatial cognitive \& logical reasoning ( N3) } \\
\text { Spatial perception ability (N4) } \\
\text { Judgment \& Decision making (N5) } \\
\text { Working memory \& attention ( N6) }\end{array}$ & Flight cognitive Capability Test \\
\hline Emotion health (E1) & $\begin{array}{l}\text { Depression (DEP), Anxiety (ANX), Hostility } \\
\text { (HOS), Phobic Anxiety (PHOB) }\end{array}$ \\
\hline Stress situation (E2) & $\begin{array}{l}\text { Somatization (SOM), Paranoid Ideation } \\
\text { (PAR), Obsessive Compulsive (O-C), }\end{array}$ \\
\hline Adaptability (E3) & $\begin{array}{l}\text { Interpersonal Sensitivity (I-S), Psychoticism } \\
\text { (PSY), Positive Symptom Distress Index } \\
\text { (PSDI) }\end{array}$ \\
\hline
\end{tabular}




\section{Data Analysis and Results}

\subsection{Model Modifying and Evaluation}

SEM is a kind of linearity modeling tool using statistical data, which is suitable for the problem with unobservable variables especially $[9,10]$. The modeling criterion is as follows. First, the smaller the ratio of chi-square and the degrees of freedom is, the better the goodness between model and practice is; second, P-value is used to test the significance level of model variables, whose value should be less than 0.1 ; third, the value of Comparative Fit Index (CFI , Incremental Fit Index (IFI) and goodness of fit index(GFI) should all be greater than 0.9, while the value of Standardized Root Mean Square Residua (SRMR) should be less than 0.08 .

The model is iteratively modified according to the modification indices obtained from the model itself until the estimating value is suited for the requirement of criterion. After the iterative process of testing and modifying, the goodness-of-fit statistics of the primitive model and the final revised model are shown in Table 5.

Table 5. Goodness of fit statistics

\begin{tabular}{cccc}
\hline Goodness of fit statistics & primitive model & final model & Recommended values for fit \\
\hline CMIN/DF & 1.535 & 1.296 & $<=2$ \\
IFI & 0.825 & 0.907 & $>0.9$ \\
GFI & 0.656 & 0.696 & $>0.9$ \\
CFI & 0.818 & 0.903 & $>0.9$ \\
RMSEA & 0.091 & 0.068 & $<0.08$ \\
SRMR & 0.1024 & 0.0955 & $<0.08$ \\
PGFI & 0.554 & 0.568 & $>0.5$ \\
PNFI & 0.559 & 0.601 & $>0.5$ \\
\hline
\end{tabular}

\subsection{Results}

Table 6 lists the results for the hypotheses. After deleting those invalid paths, the internal relationship will be easily found with the help of those paths showed in Fig.2.

This model is proved to be higher goodness of fit in relationship among three dimensions than the original one. The SEM model shows that both personality traits and mental health have an effect on the cognitive functions. All sub-dimensions of personality traits expect for intellect have an effect on pilots' basic cognitive abilities by affecting their mental health states. Each of the three sub-dimensions of mental health has a significant effect on the basic cognitive abilities of pilots; emotion characteristics, character traits and working attitude all have significantly direct effects on the adaptability in mental health; however, the direct influence of intellect on mental health is not significant. In addition, the intellect in personality traits has a great 
direct influence on the basic flight cognitive abilities. Meanwhile, character traits dramatically affect comprehension, attention and so on. Spatial sense, comprehension, attention and working memory are affected significantly by emotion health and stress level of a pilot's mental health.

Table 6. Results of hypotheses testing

\begin{tabular}{lcccc}
\hline H\# & Relationship & P-value & & Support \\
\hline H1 & N1<--P2 & 0.411 & & NO \\
H2 & N2<--P2 & 0.011 & $\mathrm{p}<0.05$ & YES \\
H3 & N3<--P2 & 0.821 & & NO \\
H4 & N4<--P2 & 0.045 & $\mathrm{p}<0.05$ & NO \\
H5 & N5<--P2 & 0.169 & & NO \\
H6 & N6<--P2 & 0.748 & & NO \\
H7 & N2<--P3 & 0.398 & & NO \\
H8 & N5<--P3 & 0.366 & & NO \\
H9 & N1<--P4 & 0.015 & $\mathrm{p}<0.05$ & YES \\
H10 & N2<--P4 & 0.008 & $\mathrm{p}<0.01$ & YES \\
H11 & N1<--E3 & 0.813 & & NO \\
H12 & N2<--E3 & 0.063 & $\mathrm{p}<0.1$ & NO \\
H13 & N3<--E3 & 0.795 & & NO \\
H14 & N4<--E3 & 0.009 & $\mathrm{p}<0.01$ & YES \\
H15 & N5<--E3 & 0.038 & $\mathrm{p}<0.05$ & NO \\
H16 & N6<--E3 & 0.778 & & NO \\
H17 & N1<--E2 & 0.061 & $\mathrm{p}<0.1$ & YES \\
H18 & N3<--E2 & 0.801 & & NO \\
H19 & N6<--E2 & 0.739 & & NO \\
H20 & N1<--E1 & 0.036 & $\mathrm{p}<0.05$ & YES \\
H21 & N2<--E1 & 0.046 & $\mathrm{p}<0.05$ & YES \\
H22 & N5<--E1 & 0.033 & $\mathrm{p}<0.05$ & YES \\
H23 & N6<--E1 & $* * *$ & $\mathrm{p}<0.001$ & YES \\
H24 & E3<--P1 & 0.009 & $\mathrm{p}<0.01$ & NO \\
H25 & E3<--P3 & 0.01 & $\mathrm{p}<0.05$ & YES \\
H26 & E3<--P4 & 0.001 & $\mathrm{p}<0.01$ & NO \\
H27 & E1<--P1 & 0.443 & & NO \\
H28 & E2<--P4 & 0.768 & & NO \\
\hline
\end{tabular}

$* * *$ stand for significant difference $(\mathrm{p}<0.001)$. 


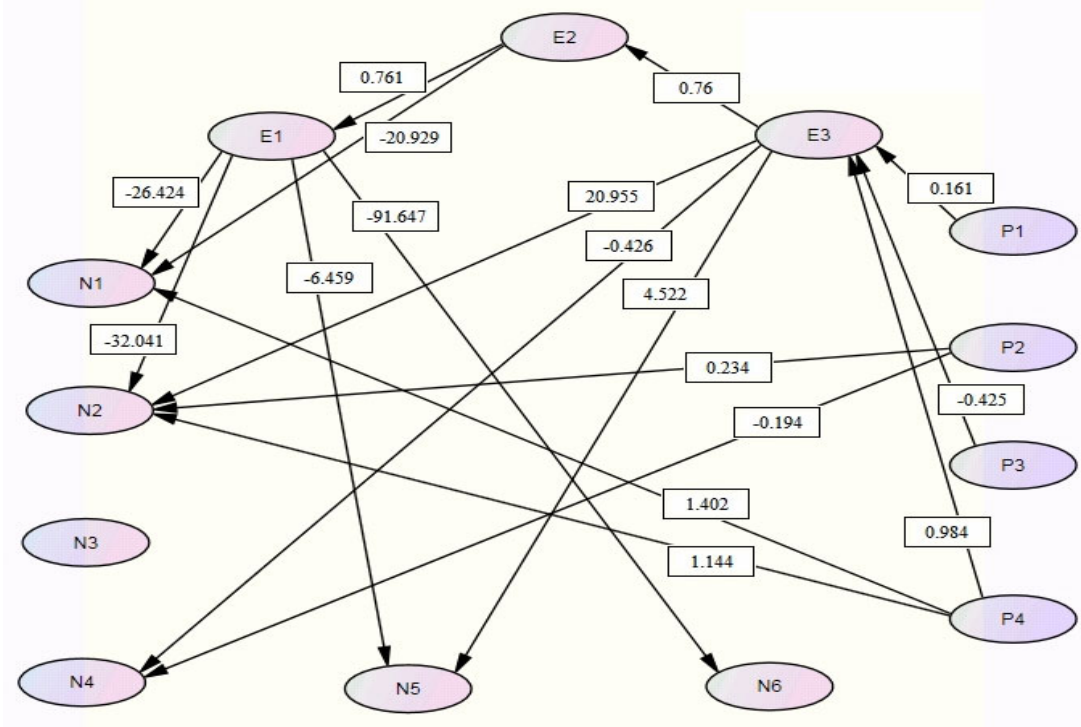

Fig. 2. Structural equation model of the study

\subsection{Discussions}

Theoretically, cognitive functions should be affected directly by personality traits. The model has shown that emotion characteristics, character traits and working attitude have effects on a pilot's basic cognitive ability through the way of affecting his mental health state. Emotion characteristics are recognized as emotional stability and the trend of mental experiences. Pilots in an instability mode tend to experience tension, worry and a lack of safety more often and to have mental issues more easily than those in a stability mode. According to Eysenck's awakening theory [11, 12], individuals have a high level of arousal to get the motivation to finish tasks in an emergency, which makes anxiety and stress more easily and affects cognitive functions further. Character traits are important for the development of positive emotion, good interpersonal skills and avoidance of mental problems. Past studies have found that most of the pilots have the extroversion personality [13, 14]. Researchers have become much aware of the importance of effective communication in multi-operator environments to support team performance [15]. In a high-risk situation, crew interactions with exact information and consentient situation perception can generate better decisions to solve problem compared to that of any individual. Working attitude not only directly affects spatial cognitive and speech ability, but also has impacts on a pilot's basic cognitive functions through the way of affecting his mental health. Pilots who have the most positive attitudes towards their job perform slightly better due to positive emotion and good adaptability. Meanwhile, intellect directly affects almost every aspect of cognitive functions. In particular, intellect has a more significant influence on speech and spatial ability than judgment and decision making. Furthermore, intellect has a more significant relationship with crystallized intelligence than fluid intelligence. 
Moreover, it is necessary to add some more paths to the model according to the modifying results. These newly added paths further explain the relationship among stress, emotion health and adaptability. Persons with good adaptability can deal with stress problems on their merits. These people are characterized by self-assurance, extroversion, and optimism. It is easy for negative emotions to appear when something unusual takes place during their work or life. It has been proven that high workload and high risk of flight work lead pilots to experience stress more often and more intensely than people holding other jobs. At the same time, a negative emotion arises from extreme stress, and it could further affect various cognitive functions.

\section{Conclusions}

This study has proven again that not only flight cognitive abilities have impacts on pilots in terms of their flight performances, but also personality traits and mental health do so. In particular, interpersonal relationships, stress, emotional stability etc. have significant influences on a pilot's cognitive performance. Moreover, the relationship model has also shown that personality traits have an increasing effect on the cognitive operations with the increase of task difficulty and stress. Meanwhile, factors such as individual anxiety, depression and impulsivity also affect a pilot's flight performance. Furthermore, the research provides a tool to explore the relationship between pilots and their operational traits via QAR (Quick Access Recorder) data, which is useful for managing pilots' human errors.

\section{References}

1. Dockeray, F.C., Isaacs, S.: Psychological research in aviation in Italy, France, England, and the American Expeditionary Forces. Journal of Comparative Psychology 1, 115-148 (1921)

2. Sells, S.B.: Development of a personality test battery for psychiatric screening of flying personnel. Journal of Aviation Medicine 26, 35-45 (1955)

3. Sells, S.B.: Further developments on adaptability screening for flying personnel. Aviation Medicine 27, 440-451 (1956)

4. Hunter, D.R., Burke, E.F.: Handbook of pilot selection. Avebury Aviation, Aldershot (1995)

5. Hunter, D.R., Martinussen, M.: Aviation Psychology and Human Factor. CRC Press, Boca Raton (2010)

6. Lang-Ree, O.C., Martinussen, M.: Applicant reactions and attitudes towards the selection procedure in the Norwegian Air Force. Human Factors and Aerospace Safety 6, 345-358 (2006)

7. Orasanu, J.: Stress and naturalistic decision making: Strengthening the weak links. Ashgate, Aldershot (1997)

8. Simon, H.: Causal ordering and identifiability. In: Hood, W.C., Koopmans, T.C. (eds.) Studies in Econometric Method, pp. 49-74. Wiley, New York (1953)

9. Boucard, A., Marchand, A., Noguès, X.: Reliability and validity of structural equation modeling applied to neuroimaging data: A simulation study. Journal of Neuroscience Methods 166(2), 278-292

10. Jietai, H., Zhonglin, W., Zijun, C.: Structural equation model and its application. Educational Science Publishing House, Beijing (2005) 
11. Eysenek, H.J., Eysenek, M.W.: Personality and individual differences. Plenum, NewYork (1985)

12. Eysenck, H.J.: Relation between intelligence and personality. Perceptual and Motor Skills 32, 637-638 (1971)

13. Xiaowei: The research on the personality traits influencing flying cadets emotional stability. Department of Psychology Faculty of Aerospace Medicine, The Fourth Military Medical University, Xi' an (2002)

14. Yu, H., Jiao, Z.: A study of personality characteristics in pilots in the civil aviation. Shandong Arch. Psychiatry 19(2), 113-115 (2006)

15. Harris, D., Muir, H.C.: Contemporary issues in human factors and aviation safety. United Kingdom, Surrey, Ashgate (2005)

16. Eysenck, H.J.: Personality and intelligence: psychometric and experimental approaches. In: Sternberg, R.J. (ed.) Personality and Intelligence, pp. 3-31. Cambridge University, New York (1994)

17. McCann, S.J.H.: Emotional Health and the Big Five Personality Factors at the American State Level. Cape Breton University, Sydney

18. Liu, Y., Gordon-Becker, S.E.: An introduction to human factors engineering. Pearson Education, New Jersey (2004)

19. Ellis, B.B., Mead, A.D.: Assessment of the Measurement Equivalence of a Spanish Translation of the 16PF Questionnaire. In: Educational and Psychological Measurement, vol. 60(5), pp. 787-807. Sage Publications, Inc., Thousand Oaks (2004)

20. Chernyshenko, O.S., Stark, S., Chan, K.Y.: Investigating the Hierarchical Factor Structure of the Fifth Edition of the 16PF: an Application of the Schmid-Leiman Orthogonalization Procedure. In: Educational and Psychological Measurement, vol. 61(2), pp. 290-302. Sage Publications, Inc., Thousand Oaks (2001)

21. Hu, S.-J., Jou, S.-Y., Liu, Y.-H.: Structural equation model for brand image measurement of Jeans. In: Ninth International Conference on Hybrid Intelligent Systems, pp. 85-94 (2009)

22. Liu, T.-Z., Li , Z.-X.: Structural Equation Model for the Affecting Factors of Safety management Capability of Coal Mine. In: 2008 International Workshop on Modeling, Simulation and Optimization, pp. $74-77$ (2008) 